\title{
Performance and Scalability of the NAS Parallel Benchmarks in Java
}

\author{
Michael A. Frumkin, Matthew Schultz, Haoqiang Jin, and Jerry Yan \\ NASA Advanced Supercomputing (NAS) Division \\ NASA Ames Research Center, Moffett Field, CA 94035-1000 \\ \{frumkin, $\mathrm{hj}$ in, yan\}@nas.nasa.gov
}

\begin{abstract}
Several features make Java an attractive choice for scientific applications. In order to gauge the applicability of Java to Computational Fluid Dynamics (CFD), we have implemented the NAS Parallel Benchmarks in Java. The performance and scalability of the benchmarks point out the areas where improvement in Java compiler technology and in Java thread implementation would position Java closer to Fortran in the competition for scientific applications.
\end{abstract}

\section{Introduction}

The portability, expressiveness, and safety of the Java language, supported by rapid progress in Java compiler technology, have created an interest in the High Performance Computing (HPC) community to evaluate Java on computationally intensive problems [8]. Java threads, RMI, and networking capabilities position Java well for programming on Shared Memory Parallel (SMP) computers and on computational grids. On the other hand issues of safety, lack of light weight objects, intermediate byte code interpretation, and array access overheads create challenges in achieving high performance for Java codes. The challenges are being addressed by work on implementation of efficient Java compilers [10] and by extending Java with classes implementing the data types used in HPC [9].

In this paper, we describe an implementation of the NAS Parallel Benchmarks (NPB) [1] in Java. The benchmark suite is accepted by the HPC community as an instrument for evaluating performance of parallel computers, compilers, and tools. Our implementation of the NPB in Java is derived from the optimized NPB2.3-serial version [6] written in Fortran (except IS, written in C). The NPB2.3-serial version was previously used for the development of the HPF [3] and OpenMP [6] versions of the NPB. We start with an evaluation of Fortran to Java conversion options by comparing performance of basic Computational Fluid Dynamics (CFD) operations. The most efficient options are then used to translate Fortran to Java. We then parallelize the resulting code by using Java threads and the master-workers load distribution model. Finally, we profile the benchmarks and analyze the performance on five different machines: IBM p690, SGI Origin2000, SUN Enterprise10000, Intel Pentium-III based PC, and Apple G4 Xserver. The implementation will be available as the NPB3.0-JAV package from www.nas.nasa.gov.

\section{The NAS Parallel Benchmarks}

The NAS Parallel Benchmarks (NPB) were derived from CFD codes [1]. They were designed to compare the performance of parallel computers and are recognized as a standard indicator of computer performance. The NPB suite consists of three simulated CFD applications and five kernels. The simulated CFD applications use different implicit algorithms to solve 3-dimensional (3-D) compressible Navier-Stokes equations which mimic data traffic and computations in full CFD codes. The five kernels represent computational cores of numerical methods routinely used in CFD applications. 
BT is a simulated CFD application which uses an Alternating Direction Implicit (ADI) approximate factorization to decouple the $x, y$, and $z$ dimensions. The resulting system is Block Tridiagonal of $5 \times 5$ blocks is solved sequentially along each dimension. SP is a simulated CFD application which employs the Beam-Warming approximate factorization. The resulting system of Scalar Pentadiagonal linear equations is solved sequentially along each dimension. $\mathbf{L U}$ is the third simulated CFD application. It uses the symmetric successive over-relaxation (SSOR) method to solve the discrete Navier-Stokes equations by splitting it into block Lower and Upper triangular systems. FT contains the computational kernel of a 3-D Fast Fourier Transform (FFT). MG uses a V-cycle Multi Grid method to compute the solution of the 3-D scalar Poisson equation. CG uses a Conjugate Gradient method to compute approximations to the smallest eigenvalues of a sparse unstructured matrix. IS performs sorting of integer keys using a linear-time Integer Sorting algorithm based on computation of the key histogram.

\section{Fortran to Java Translation}

We are focused on achieving high performance of the code, hence we opted to do a literal translation of Fortran to Java at the procedural level and we did not attempt to convert the benchmarks into an object-oriented code. In order to compare efficiency of different options in the literal translation and to form a baseline for estimation of the quality of the benchmark translation, we chose a few basic CFD operations and implemented them in Java.

- loading/storing array elements;

- filtering an array with a local kernel; (the kernel can be a first or second order star stencil as in BT, SP, and $\mathrm{LL}^{-}$, or a compact $3 \times 3 \times 3$ stencil as in the smoothing operator in $\mathrm{MG}$ );

- a multiplication of a 3-D array of $5 \times 5$ matrices by a 3-D array of 5-D vectors; (a routine CFD operation);

- a reduction sum of 4-D array elements.

We implemented these operations in two ways: by using linearized arrays and by preserving the number of array dimensions. The version that preserves the array dimension was 1.5-2 times slower than the linearized version on the SGI Origin2000 (Java 1.1.8) and on the SUN Enterprise10000 (Java 1.1.3). So we decided to translate Fortran arrays into linearized Java arrays. The performance of the serial and multithreaded versions are compared with the Fortran version, Table 1.

Table 1. The execution times in seconds of the basic CFD operations on the SGI Origin2000. The grid size is $81 \times 81 \times 100$, the matrices are $5 \times 5$, and vectros are 5 -D.

\begin{tabular}{|l|r|r|r|r|r|r|r|r|}
\hline \hline & \multicolumn{7}{|c|}{ J77 } & \multicolumn{6}{|c|}{ Java 1.1.8 } \\
\hline & \multicolumn{2}{|c|}{} & \multicolumn{6}{|c|}{ Number of Threads } \\
\hline Operation & \multicolumn{2}{|c|}{ Serial } & 1 & 2 & 4 & 8 & 16 & 32 \\
\hline \hline 1. Assignment (10 iterations) & 0.327 & 1.087 & 1.256 & 0.605 & 0.343 & 0.264 & 0.201 & 0.140 \\
\hline 2. First Order Stencil & 0.045 & 0.373 & 0.375 & 0.200 & 0.106 & 0.079 & 0.055 & 0.061 \\
\hline 3. Second Order Stencil & 0.046 & 0.571 & 0.572 & 0.289 & 0.171 & 0.109 & 0.082 & 0.072 \\
\hline 4. Matrix vector multiplication & 0.571 & 4.928 & 6.178 & 3.182 & 1.896 & 1.033 & 0.634 & 0.588 \\
\hline 5. Reduction Sum & 0.079 & 0.389 & 0.392 & 0.201 & 0.148 & 0.087 & 0.063 & 0.072 \\
\hline
\end{tabular}

We can offer some conclusions from the profiling data:

- Java serial code is a factor of 3.3 (Assignment) to 12.4 (Second Order Stencil) slower than the corresponding Fortran operations;

- Java thread overhead ( 1 thread versus serial) contributes no more than $20 \%$ to the execution time;

- The speedup with 16 threads is around 7 for the computationally expensive operations (2-4) and is around 5-6 for less intensive operations ( 1 and 5). 
For a more detailed analysis of the basic operations we used an SGI profiling tool called perfex. The perfex uses 32 hardware counters to count issued/graduated integer and floating point instructions, load/stores, primary/secondary cache misses, etc. The profiling with perfex shows that the Java/Fortran performance correlates well with the ratio of the total number of executed instructions in these two codes. Also, the Java code executes twice as many floating point instructions as the Fortran code, confirming that the Just-In-Time (JIT) compiler does not use the "madd" instruction.

\section{Using Java Threads for Parallelization}

A significant appeal of Java for parallel computing stems from the presence of threads as part of the Java language. On a shared memory multiprocessor, the Java Virtual Machine (JVM) can assign different threads to different processors and speed up execution of the job if the work is well divided among the threads. Conceptually, Java threads are close to OpenMP threads, so we used the OpenMP version of the benchmarks [6] as a prototype for the multithreading.

The base class (and, hence, all other classes) of each benchmark was derived from java. lang. Thread, so all benchmark objects are implemented as threads. The instance of the main class is designated to be the master that controls the synchronization of the workers. The workers are switched between blocked and runnable states with wait() and notify() methods of the Thread class. The details of our translation method and the resulting code structure can be found in [4].

\section{Performance and Scalability}

We have tested the benchmarks on the classes $\mathrm{S}, \mathrm{W}$, and $\mathrm{A}$; the performance is shown for class $\mathrm{A}$ as the largest of the tested classes. The tests were performed on three large SMP machines: IBM p690 $(1.3 \mathrm{GHz}, 32$ processors, Java 1.3.0), SGI Origin2000 (250 MHz, 32 processors, Java 1.1.8), and SUN Enterprise $10000(333 \mathrm{MHz}, 16$ processors, Java 1.1.3). On the SUN Enterprise we also tested Java 1.2.2, but its scalability was worse than that of Java 1.1.3. The performance results are summarized in Tables 2-4. For comparison, we include Fortran-OpenMP results in Tables 2 and 3. For a reference we ran the benchmarks on a Linux PC (933 MHz, 2 PIII processors, Java 1.3.0) and on 1 node of Apple Xserver (1GHz, 2 G4 processors, Java 1.3.1), Tables 5,6.

Table 2. Benchmark times in seconds on IBM p690 ( $1.3 \mathrm{GHz}, 32$ processors).

\begin{tabular}{|l|r|r|r|r|r|r|r|}
\hline & & \multicolumn{6}{|c|}{ Number of Threads } \\
\hline & Serial & 1 & 2 & 4 & 8 & 16 & 32 \\
\hline \hline BT.A Java1.3.0 & 511.5 & 614.4 & 307.1 & 160.2 & 80.8 & 41.5 & 22.4 \\
\hline BT.A f77-OpenMP & 161.4 & 254.1 & 129.5 & 66.1 & 34.8 & 17.6 & 10.5 \\
\hline \hline SP.A Java1.3.0 & 407.1 & 427.5 & 214.2 & 111.1 & 58.7 & 30.8 & 33.2 \\
\hline SP.A f77-OpenMP & 142.6 & 141.2 & 72.3 & 37.8 & 18.7 & 10.2 & 6.2 \\
\hline \hline LU.A Java1.3.0 & 615.9 & 645.8 & 322.5 & 168.5 & 90.5 & 46.2 & 28.5 \\
\hline LU.A f77-OpenMP & 144.0 & 145.9 & 70.0 & 32.9 & 16.7 & 8.9 & 6.0 \\
\hline \hline FT.A Java1.3.0 & 54.4 & 46.9 & 28.8 & 15.0 & 8.6 & 5.61 & 4.83 \\
\hline FT.A f77-OpenMP & 10.8 & 11.0 & 5.5 & 2.7 & 1.4 & 0.76 & 0.55 \\
\hline \hline IS.A Java1.3.0 & 1.60 & 1.70 & 1.04 & 0.83 & 0.76 & 0.79 & 2.50 \\
\hline IS.A C-OpenMP & 1.36 & 1.87 & 1.02 & 0.55 & 0.35 & 0.27 & 0.40 \\
\hline \hline CG.A Java1.3.0 & 8.75 & 8.16 & 4.55 & 2.44 & 1.50 & 1.37 & 1.79 \\
\hline CG.A f77-OpenMP & 6.22 & 6.21 & 3.13 & 1.64 & 0.83 & 0.41 & 0.46 \\
\hline \hline MG.A Java1.3.0 & 14.55 & 14.44 & 7.76 & 4.15 & 2.39 & 1.80 & 1.70 \\
\hline MG.A f77-OpenMP & 6.95 & 6.84 & 3.34 & 1.56 & 0.86 & 0.55 & 0.44 \\
\hline
\end{tabular}


Table 3. Benchmark times in seconds on SGI Origin2000 (250 MHz, 32 processors).

\begin{tabular}{|l|r|r|r|r|r|r|r|}
\hline & & \multicolumn{7}{|c|}{ Number of Threads } \\
\hline & Serial & 1 & 2 & 4 & 8 & 9 & 16 \\
\hline \hline BT.A Java 1.1.8 & 9136.3 & 8332.5 & 4806.0 & 2645.7 & 1413.7 & 1278.0 & 838.4 \\
\hline BT.A f77-OpenMP & 1028.0 & 983.6 & 519.5 & 275.4 & 143.7 & 133.0 & 81.4 \\
\hline \hline SP.A Java 1.1.8 & 7137.4 & 7111.0 & 3789.8 & 2333.8 & 1705.2 & 1581.2 & 1188.2 \\
\hline SP.A f77-OpenMP & 944.7 & 850.8 & 504.5 & 259.9 & 147.6 & 133.0 & 88.5 \\
\hline \hline LU.A Java 1.1.8 & 9686.8 & 9967.4 & 5600.9 & 3475.8 & 2247.8 & - & 1502.2 \\
\hline LU.A f77-OpenMP & 1104.8 & 926.9 & 439.7 & 236.4 & 132.5 & 121.1 & 75.72 \\
\hline \hline FT.A Java 1.1.8 & 656.0 & 630.8 & 361.1 & 174.9 & 110.6 & - & 63.8 \\
\hline FT.A f77-OpenMP & 82.3 & 74.8 & 41.1 & 21.1 & 11.7 & 10.9 & 7.1 \\
\hline \hline IS.A Java 1.1.8 & 10.7 & 12.7 & 15.0 & 17.9 & 11.8 & - & 17.9 \\
\hline IS.A C-OpenMP & 4.9 & 4.9 & 2.7 & 1.5 & 1.0 & - & 0.9 \\
\hline \hline CG.A Java 1.1.8 & 105.0 & 112.4 & 114.2 & 53.9 & 52.6 & - & 23.1 \\
\hline CG.A f77-OpenMP & 39.7 & 35.6 & 21.8 & 10.2 & 3.6 & 3.2 & 2.7 \\
\hline \hline MG.A Java 1.1.8 & 254.0 & 263.7 & 189.3 & 108.4 & 70.8 & - & 45.0 \\
\hline MG.A f77-OpenMP & 36.4 & 36.8 & 23.0 & 12.7 & 7.7 & 6.4 & 4.1 \\
\hline \hline
\end{tabular}

Table 4. Benchmark times in seconds on SUN Enterprise10000 (333 MHz, 16 processors).

\begin{tabular}{|l|r|r|r|r|r|r|r|r|}
\hline & & \multicolumn{7}{|c|}{ Number of Threads } \\
\hline & Serial & 1 & 2 & 4 & 8 & 9 & 12 & 16 \\
\hline \hline BT.A Java1.1.3 & 13609.5 & 14671.3 & 7381.7 & 3846.3 & 2305.0 & 2042.7 & 1782.7 & 1762.2 \\
\hline SP.A Java1.1.3 & 10235.8 & 11108.1 & 5692.9 & 3409.3 & 2095.5 & 1899.1 & 1862.1 & 1671.2 \\
\hline LU.A Java1.1.3 & 12344.5 & 13578.9 & 6843.3 & 3765.7 & 2077.3 & 1892.7 & 1730.2 & 1745.4 \\
\hline FT.A Java1.1.3 & 1104.6 & 1318.8 & 674.7 & 384.2 & 342.7 & - & 353.4 & 363.3 \\
\hline IS.A Java1.1.3 & 22.9 & 29.4 & 15.7 & 9.0 & 8.4 & - & 8.9 & 13.6 \\
\hline CG.A Java1.1.3 & 203.8 & 215.3 & 111.6 & 69.0 & 47.6 & - & 40.8 & 36.4 \\
\hline MG.A Java1.2.2 & 438.9 & 494.7 & 244.8 & 138.5 & 87.1 & - & 72.6 & 68.7 \\
\hline \hline
\end{tabular}

\subsection{Comparison to Fortran Performance}

We offer the following conclusions from the performance results. There are two groups: benchmarks BT, SP, LU, FT, and MG working on structured grids; and benchmarks IS and CG involving unstructured computations. For the first group, on the Origin 2000 the serial Java/Fortran execution time ratio is within the interval 8.3-10.8, which is within the 8.2-12.5 intervals for the computationally intensive basic CFD operations, Table 1, indicating that our implementation of the benchmarks adds little performance overhead to the overhead of the basic operations. On the p690 the ratio for this group is within interval 2.1-5.1. For the second group, the Java/Fortran execution time ratio is within the 3.11-7.2 and 1.1-1.3 interval for Origin 2000 and p690 respectively. The separation into two groups may be explained by the fact that the $f 77$ compiler optimizes regular-stride computations much better than the Java compilers.

The benchmarks working on structured grids heavily involve the basic CFD operations and any performance improvement of the basic operations would directly affect performance of the benchmarks. Such improvement can be achieved in three ways. First, JIT needs to reduce the ratio of Java/Fortran instructions (which for Origin 2000 is a factor of 10) for executing the basic operations. Second, the Java rounding error model should allow the "madd" instruction to be used. Third, in the codes where the array sizes and loop bounds are constants, a compiler optimization should be able to lift bounds checking out of the loop without compromising code safety [8].

Our performance results apparently are in sharp contrast with the results of the Java Grande Benchmarking Group [2] reporting that on almost all Java Grande Benchmarks, the performance of a Java version is within a 
Table 5. Benchmark times in seconds on Linux PC (933 MHz, 2 PIII processors).

\begin{tabular}{|l|r|r|r|}
\hline \hline & & \multicolumn{2}{|c|}{ Number of Threads } \\
\hline Java1.3.0 & Serial & 1 & 2 \\
\hline \hline BT.A & 8007.8 & 8007.7 & 8083.2 \\
\hline SP.A & 3543.9 & 4198.7 & 4201.9 \\
\hline LU.A & 5887.9 & 7151.7 & 7140.7 \\
\hline FT.A & 411.0 & 493.0 & 494.4 \\
\hline IS.A & 9.1 & 9.4 & 9.8 \\
\hline CG.A & 116.8 & 75.8 & 77.0 \\
\hline MG.A & 195.0 & 170.3 & 188.2 \\
\hline
\end{tabular}

Table 6. Benchmark times in seconds on Apple Xserver (1 GHz, 2 G4 processors).

\begin{tabular}{|l|r|r|r|}
\hline \hline & & \multicolumn{2}{|c|}{ Number of Threads } \\
\hline Java1.3.0 & Serial & 1 & 2 \\
\hline \hline BT.A & 2043.15 & 2120.87 & 1185.97 \\
\hline SP.A & 1377.56 & 1487.30 & 845.91 \\
\hline LU.A & 17779.24 & 19075.83 & 9883.85 \\
\hline FT.A & 179.71 & 161.31 & 95.93 \\
\hline IS.A & 7.08 & 7.59 & 6.00 \\
\hline CG.A & 51.62 & 48.69 & 28.08 \\
\hline MG.A & 59.94 & 60.12 & 36.07 \\
\hline \hline
\end{tabular}

factor of 2 of the corresponding $\mathrm{C}$ or Fortran versions. To resolve the performance gap we obtained the jgf_2.0 from the www.epcc.ed.ac.uk/java_grande website. Since the Fortran version was not available on the website we literally translated the Java version to Fortran and ran both versions on multiple platforms, Table 7. We have also included results of the LINPACK version of the LU decomposition. From the table we can conclude that the algorithm used in lufact benchmark performs poorly relative to LINPACK. The reason for this is that lufact is based on BLAS1, having poor cache reuse. As a result, the computations always wait for data (cache misses), which obscures the performance comparison between Java and Fortran. Note that our Assignment base operation exhibits about the same Java/Fortran performance ratio as the lufact benchmark.

Table 7. Java Grande LU benchmark [2]. The Fortran version was directly derived from Iufact. The performance of the LINPACK version of the LU decomposition (DGETRF, based on MMULT, and having good cache reuse) is shown for reference. The classes $\mathrm{A}, \mathrm{B}$ and $\mathrm{C}$ employ $500 \times 500,1000 \times 1000$ and $2000 \times 2000$ matrices respectively. The execution time is in seconds.

\begin{tabular}{|l|r|r|r|r|r|r|r|r|r|}
\hline & \multicolumn{3}{|c|}{ Java } & \multicolumn{3}{|c|}{ f77 } & \multicolumn{3}{|c|}{ LINPACK } \\
\hline \hline Machine/Platform & $\mathrm{A}$ & $\mathrm{B}$ & $\mathrm{C}$ & $\mathrm{A}$ & $\mathrm{B}$ & $\mathrm{C}$ & $\mathrm{A}$ & $\mathrm{B}$ & $\mathrm{C}$ \\
\hline SUN UltraSparc/Java 1.4.0 & 3.13 & 27.78 & 250.3 & 0.36 & 8.11 & 104.0 & 0.423 & 3.448 & 29.93 \\
\hline SGI Origin2000/Java 1.1.8 & 3.05 & 28.10 & 266.7 & 0.70 & 7.94 & 86.3 & 0.207 & 1.710 & 13.78 \\
\hline Sun E10000/Java 1.1.3 & 3.86 & 48.92 & 512.0 & 1.41 & 29.90 & 395.6 & 0.522 & 4.411 & 48.55 \\
\hline IBM POWER4/Java 1.3.0 & 0.27 & 2.60 & 21.3 & 0.17 & 2.19 & 17.6 & 0.031 & 0.237 & 1.74 \\
\hline
\end{tabular}

\subsection{Scalability of Multithreaded Java Codes}

Some singlethreaded Java benchmarks run faster than the serial versions. That can be explained by the fact that in these singlethreaded versions the data layout is more cache friendly. Overall the multithreading introduces an overhead of about $10 \%-20 \%$. The speedup of BT, SP, and LU with 16 threads is in the range of 6-12 (efficiency 0.38-0.75). The low efficiency of FT on SUN Enterprise is explained by the inability of the JVM to use more than 4 processors to run applications requiring significant amounts of memory (FT.A uses about $350 \mathrm{MB}$ ). An artificial increase in the memory use for other benchmarks also resulted in a drop of scalability. The lower scalability of LU can be explained by the fact that it performs the thread synchronization inside a loop over one grid dimension, thus introducing higher overhead. The low scalability of IS was expected since the amount of work performed by each thread is small relative to other benchmarks, hence, the data movement overheads eclipse the gain in processing time.

Our tests of CG (and IS) benchmark on the SGI Orgin2000 showed virtually no performance gain until 8 processors were used. Even with 10-16 requested threads, only 2-4 processors were used. To investigate this 
problem, we used "top - T" command to monitor the individual Posix threads and found that the JVM ran all the threads in 1-2 Posix threads. The fact that all the other benchmarks ran each thread in a separate Posix thread suggested that the problem was peculiar to CG. CG's work load is much smaller than the work load of the computationally intensive benchmarks and we assumed that the JVM was attempting to optimize CPU usage by running the threads serially on a few processors. In order to test this assumption, we put an initialization section performing a large work in each thread. This forced the JVM to assign all requested threads to different CPUs. When the actual computations did start, JVM indeed used a separate CPU for each thread. Hence, by initializing the thread load, we were able to get a visible speedup of CG, Table 3. On the Linux PIII PC we did not obtain any speedup on any benchmark when using 2 threads. The reason for this will be farther investigated.

\section{Related Work}

In our implementation we parallelized the NAS Parallel Benchmarks using Java threads. The Performance Engineering Group at the School of Computer Science of the University of Westminster used the Java Native Interface to create a system dependent Java MPI library. They also used this library to implement the FT and IS benchmarks using javaMPI [5].

The Distributed and High Performance Computing Group of the University of Adelaide, has also released the EP and IS benchmarks (FT, CG, and MG are under development) [7], along with a number of other benchmarks in order to test Java's suitability for grand challenge applications.

The Java Grande Forum have developed a set of benchmarks [2] reflecting various computationally intensive applications which likely will benefit from use of Java. The performance results reported in [2] relative to $\mathrm{C}$ and Fortran are significantly more favorable to Java than ours.

\section{Conclusions}

Although the performance of the implemented NAS Parallel Benchmarks in Java is lagging far behind Fortran and $\mathrm{C}$ at this time, by using the performance enhancing methods detailed in $[8,10]$, the serial performance can be improved to near Fortran-like performance. Efficiency of parallelization with threads is about 0.5 for up to 16 threads and is lower than the efficiency of parallelization with OpenMP, MPI, and HPF on SGI and SUN machines. However, on the IBM machine, the scalability of the Java code is as good as that of OpenMP, and in average the performance of the Java code is within a factor of 3 of that of Fortran. With several groups working on MPI and OpenMP for Java, improvements in parallel performance and scalability seem likely as well.

The attraction of Java as a language for scientific applications is primarily driven by its ease of use, universal portability, and high expressiveness which, in particular, allows expressing parallelism. If Java code is made to run faster through methods that have already been researched extensively, such as high order loop transformations, semantic expansion, together with an implementation of multidimensional arrays and complex numbers, it could be an attractive programming environment for HPC applications.

\section{References}

[1] D. Bailey, J. Barton, T. Lasinski, and H. Simon (Eds.). The NAS Parallel Benchmarks. NAS Technical Report RNR-91-002, NASA Ames Research Center, Moffett Field, CA, 1991, http://www . nas .nasa.gov/Sof tware/NPB/.

[2] J.M. Bull, L.A. Smith, L. Pottage, R. Freeman. Benchmarking Java against $C$ and Fortran for Scientific Applications. Joint ACM Java Grande - ISCOPE 2001 Conference, Palo Alto, CA, pp. 975-105. Tes source code: http://www.epcc.ed.ac.uk/computing/research_activities/java_grande/index_1.html.

[3] M. Frumkin, H. Jin, J. Yan. Implementation of NAS Parallel Benchmarks in High Performance Fortran. CDROM version of IPPS/SPDP 1999 Proceedings, April 12-16, 1999, San Juan, Puerto Rico, 10 pp.

[4] M. Frumkin, M. Schultz, H. Jin, J. Yan. Implementation of NAS Parallel Benchmarks in Java. To be published as NAS Technical Report RNR-02-XXX, NASA Ames Research Center, Moffett Field, CA, 2002, http://www.nas.nasa.gov/Software/NPB/. 
[5] V. Getov, S. Flynn-Hummel, S. Mintchev. High-Performance Parallel Programming in Java: Exploiting Native Libraries. Proceedings of the 1998 ACM Workshop on Java for High-Performance Network Computing, 10 pp., http://perun.hscs.wmin.ac.uk/JavaMPI.

[6] H. Jin, M. Frumkin, J. Yan. The OpenMP Implementation of NAS Parallel Benchmarks and Its Performance. NAS Technical Report RNR-99-011, NASA Ames Research Center, Moffett Field, CA, 1999, http://www nas.nasa.gov/Software/NPB/.

[7] J.A. Mathew, P.D. Coddington and K.A. Hawick. Analysis and Development of Java Grande Benchmarks. Proc. of the ACM, 1999 Java Grande Conference, San Francisco, June 1999, 9 pp., http://www.cs.ucsb.edu/conferences/java99/program.html.

[8] S.P. Midkiff, J.E. Moreira, M. Snir. Java for Numerically Intensive Computing: from Flops to Gigaflops. Proceedings of FRONTIERS'99, pp. 251-257, Annapolis, Maryland, February 21-25, 1999.

[9] P. Wu, S. Midkiff, J. Moreira and M. Gupta. Efficient Support for Complex Numbers in Java. Proc. of the ACM 1999 Java Grande Conference, San Francisco, June 1999, 10 pp., http://www.cs.ucsb.edu/conferences/java99/program.html.

[10] J. E. Moreira, S. P. Midkiff, M. Gupta, P. V. Artigas, M. Snir, and R. D. Lawrence. Java programming for high-performance numerical computing. IBM Systems Journal, Vol. 39, n. 1, 2000. http://www.research.ibm.com/journal/sj/391/moreira.html. 